\title{
Does the ideal health care system exist? Will it be accepted in Canada?
}

\author{
Jugpal S Arneja MD MBA FRCSC ${ }^{1}$, Edward W Buchel MD FACS, Editor-in-Chief ${ }^{2}$
}

\begin{abstract}
Tet's face it, surgery is expensive, resource intensive and complex. LIn most Canadian provinces, health care has assumed $>50 \%$ of the overall provincial budget and these costs show no signs of falling. On a cursory count, at least one dozen separate processes need to dovetail for a surgical procedure to occur, including a suitable infrastructure (preoperative, operative, recovery), appropriate equipment, adequate human resources (nursing, anesthesia, surgery and support staff) and aligned patient variables (correct indication, informed consent, fasted, etc). In the United States, health systems leverage the operating room as a profit centre, passing these costs to the insurer, while in Canada (and other socialized health care systems), the operating room is the most expensive cost centre in any facility. Currently, the United States leads the world in health care spending, which has surpassed $17 \%$ of gross domestic product (GDP). Although not as high in Canada, health care spending was approaching $12 \%$ of GDP. Throughout the world, many surgical techniques have been standardized, with reportable standardized outcomes related to the specific surgery. Little comparative data are available to evaluate the system providing the resources for the reconstructive surgeon and their patients. As these health delivery systems evolve, the question as to what is ideal continually arises.
\end{abstract}

\section{THE CURRENT CANADIAN PERSPECTIVE}

Proud politicians in Canada will tout the universality associated with the Canada Health Act. In theory, every resident has the ability to access the system; however, lengthy wait lists for elective diagnostics and interventions limit true universality. Furthermore, geographical differences, exclusions for specific covered services and differences in provincial health care plans significantly limit two other components of the Canada Health Care Act: portability and comprehensiveness. Patients know no different system. Since the initiation of the current system in 1984, a full generation of patients have become resigned to the fact that they may wait several months for a specialist appointment, specialized diagnostics and elective surgery.

Given the per capita dearth of physicians and medical centres in Canada, there is virtually no competition in the health care system for patients. Within a few months of starting a surgical practice, a surgeon's clinics are full and a wait list is initiated for surgery. The 'need' for physicians or hospitals to provide excellent care to recruit patients does not exist in any real sense.

On the insurance payment end, each provincial government is essentially a single payer, setting fee tariffs and allowing access to resources. Although reimbursements are lower per case compared with the United States, Canadian surgeons are reimbursed in a relatively consistent and reliable manner. Medical malpractice is also a fraction of the cost compared with our American colleagues. This is primarily due to the absence of a personal injury/medicolegal culture in Canada, likely driven by tort laws that prevent dramatic settlements.

\section{THE AMERICAN MODEL}

Health care in the United States is managed my multiple regional private or public insurers, and the administration of these services differs from state to state. Complexities pertain to preauthorizations, claims/reimbursements, coverage networks and the fact that medical decisions for treatment are not always made by physicians. Individual practitioners often have to 'carry the cost' associated with insurance authorizations and billing/collections. Additionally, at each level of assessment and treatment, medicolegal ramifications influence diagnostic and treatment decision making. The high prevalence of malpractice claims result in high malpractice insurance rates, ranging from approximately $\$ 40,000$ to $\$ 100,000$ in the United States versus $\$ 6,200$ to $\$ 17,500$ in Canada per annum. It has been estimated that the practice of 'defensive medicine' is performed by $>90 \%$ of practitioners. While tort reform in the United States was designed to decrease frivolous malpractice claims, they may not ultimately decrease malpractice insurance rates; the indirect costs associated with a reduction in defensive medicine should ease the cost burden attributed to health care GDP in the United States.

However, for the great majority of patients, the system does offer several advantages. More plastic surgeons, more hospitals, closer patient proximity to medical care, plentiful resources with limited wait times for diagnostics and treatment lead to extensive options for patients in a timely fashion. Patients have grown to expect timely care and are more often than not well-informed consumers. With the availability of resources comes competition for patients. Although occasionally problematic, competition does provide an incentive for constant re-evaluation and improvement in care delivery. In fact, for a variety of reasons, plastic surgical care in the United States has become supra-specialized. Surgeons focusing their expertise on a particular clinical realm have become the norm compared with a more generalized practice profile in Canada. The goal is to provide the best care possible to only a subset of patients. The introduction of 'Obamacare', the Affordable Care Act, is addressing the 'universality gap' between the United States and Canada. It can now be argued that with cost control, there may be better 'value' in the American system.

\section{THE IDEAL SYSTEM?}

The burning platform question that must be asked of health care administrators and politicians is, are Canadians getting a good deal for the amount of tax dollars plus provincial health care premiums incurred annually? Canada may never evolve into a prevention-based model similar to Cuba, with low costs and impressive life expectancies, nor a health care bank account model similar to Singapore, where the patient is empowered for medical decision making. Moving to a twotiered system or blended model such as those in the United Kingdom or Australia has proven difficult politically despite many potential advantages. The idea of expanding a second tier that could offload the public system, improve access, develop efficiencies and deliver specialty care has been halted merely due to negative public perceptions with privatization of the health care system. While Canada stands still, the Affordable Health Care Act in the United States is mandating universal accessibility through guaranteed issue of insurance and minimum standards for health insurance policies, while continuing the individual mandate of the insured person. We are now witnessing the evolution of the system in the United States toward a more 'Canadian model'.

${ }^{1}$ Division of Plastic Surgery, University of British Columbia, Vancouver, British Columbia; ${ }^{2}$ Division of Plastic Surgery, University of Manitoba, Winnipeg, Manitoba

Correspondence and reprints: Dr Jugpal S Arneja, University of British Columbia, British Columbia Children's Hospital, Division of Plastic Surgery, K3-131, 4480 Oak Street, Vancouver, British Columbia V6H 3V4. Telephone 604-875-2794, fax 604-875-2749, e-mail jugpal.arneja@ubc.ca 
To evolve our current system, Canadian health care must make changes that are politically and fiscally acceptable to the entire population. Canada has a long tradition of arms-length government oversight companies in the form of Crown corporations at both the provincial and federal levels. The Canadian Broadcasting Corporation, Canada Post, Petro Canada, Manitoba Telecom Services and the Bank of Canada were established as Crown corporations, with some ultimately being spun off into fully privatized companies. Why not create provincial Crown health corporations? Such provincial entities would allow increased transparency and accountability toward quality, accessibility, fiscal awareness and prudence. Customer service could become a focus. Government oversight would still exist while at the same time decreasing the political interference that currently overruns health care systems. In the long term, the provincial Crown corporations could decide to privatize certain aspects of care through public private partnerships, thereby creating a 'marketplace' and increasing competition that would allow accrued benefits for both the system and the consumer. Crown corporations could be a politically acceptable option for advancing our health care system without completely dismantling it. 